DOI: http://dx.doi.org/10.12660/gvcasosv7n2c15

\title{
PRÁTICA INTERCULTURAL DA LIDERANÇA: DESAFIOS NA ORGANIZAÇÃO ROTARY
}

Cross-cultural practice of leadership: challenges in the Rotary organization

OTACÍLIO TORRES VILAS-BOAS - otaciliotorres@ hotmail.com

Universidade Federal da Bahia - Savador, BA, Brasil

EDUARDO DAVEL - davel.eduardo@ gmail.com

Universidade Federal da Bahia - Savador, BA, Brasil

\section{Resumo}

Este caso de ensino proporciona uma aprendizagem voltada para o conhecimento das dimensões culturais relacionadas à liderança, para o seu entendimento como expressão intercultural e para a discussão da liderança intercultural a partir da perspectiva da prática. O caso consiste na história de descobertas de João, rotariano - isto é, associado da organização Rotary - que, ao participar de um projeto internacional pela primeira vez, acompanha o trabalho dos líderes, que inclui a formação de parceria, a interação com os rotarianos envolvidos e as comunidades nas quais o projeto é realizado, e a solução de problemas. Com base na experiência vivida por João, é possível conhecer as práticas de liderança no contexto considerado, que estão associadas à presente interação entre culturas. Dessa forma, o caso possibilita que se analise e compreenda de que forma seguidores de diferentes culturas se unem aos líderes, o modo pelo qual líderes conduzem seguidores de diferentes culturas para a realização de objetivos e como são conciliadas as diferenças entre líderes e seguidores de diferentes culturas durante o seu convívio, desafios ligados ao contexto em questão.

Palavras-chave: liderança, interculturalidade, prática, projetos internacionais, Rotary

\begin{abstract}
This teaching case allows us to develop a learning process focused on the knowledge of cultural dimensions related to leadership, the understanding of leadership as a cross-cultural expression and the discussion of cross-cultural leadership from the practice perspective. The case refers to the discoveries of João, a rotarian - that is, an associate of the Rotary organization - who, by participating in an international project for the first time, follows the work of the leaders, including partnership building, interaction with rotarians involved and the communities in which the project is carried out, and problems solution. Based on João's experience, it is possible to know the leadership practices in the considered context, which are associated to the present interaction between cultures. In this way, the case makes it possible to analyze and understand how followers from different cultures join leaders, how leaders lead followers from different cultures to achieve goals and how the differences between leaders and followers from different cultures are reconciled during their period together, challenges related to the considered context.
\end{abstract}

Keywords: leadership, cross-culturality, practice, international projects, Rotary

\section{João ingressa em um clube de Rotary e vivencia a cultura da organização}

João, após convite de Maria, conheceu o clube de Rotary do qual ela fazia parte. O clube está localizado na cidade de Salvador, na qual ambos residem. Ao convidar João, Maria disse que os 
clubes de Rotary são formados por voluntários que se dedicam à realização de projetos humanitários, tendo como base o ideal de servir, que está associado ao lema da organização - "Dar de si antes de pensar em si". Maria acreditava que João se interessaria em fazer parte do grupo, o que se concretizou após o ritual padrão da organização. Para que uma pessoa torne-se integrante de um clube de Rotary, é preciso inicialmente que ela participe de reuniões do clube como convidada. Durante tal processo, ela deve receber orientações em relação ao Rotary, e espera-se que ela fique ambientada ao clube. Em seguida, caso ela deseje fazer parte do clube, seus integrantes devem aprovar o seu ingresso. Com isso, verifica-se se, na opinião daqueles que já integram o clube, a pessoa mostra-se sintonizada com os ideais do grupo. Por fim, havendo a aprovação, realiza-se a cerimônia referente a sua posse como rotariana, que inclui a leitura de um juramento. Ela passa a usar um distintivo com o emblema do Rotary, que a identifica como integrante da organização, e a pessoa que a convidou para participar do clube passa a ser seu padrinho ou sua madrinha no Rotary.

O emblema do Rotary traz uma roda denteada com 24 dentes, simbolizando que os rotarianos devem estar voltados para servir 24 horas por dia. Enquanto os rotarianos são identificados pelo uso do distintivo, os clubes de Rotary contam com artefatos que se fazem presentes em qualquer reunião de clube: sua bandeira, um sino e um martelo. Enquanto a bandeira traz o emblema do Rotary, o sino e o martelo representam disciplina e autoridade do presidente para conduzir as reuniões, sendo utilizados para iniciá-las, finalizá-las e em qualquer outro momento em que se deseje atenção.

No cotidiano como rotariano, João rapidamente notou que a "Prova Quádrupla" ("Do que nós pensamos, dizemos ou fazemos: É a verdade? É justo para todos os interessados? Criará boa vontade e melhores amizades? Será benéfico para todos os interessados?"), reflexão ligada ao servir criada em 1932 pelo rotariano Herbert Taylor (Rotary, 2017) ${ }^{1}$, é sempre citada. Também percebeu que a referência ao servir também é vista nas publicações dirigidas aos rotarianos e nos eventos do Rotary. Nos textos produzidos periodicamente na sede e nos distritos (os clubes de Rotary são agrupados em distritos), que abordam temas diversos ligados à organização, o servir é habitualmente enfatizado. Participando de eventos do Rotary, João viu que o reforço ao servir integra as palestras, apresentações de projetos e demais atividades.

\section{João e outros integrantes do clube se envolvem em um projeto internacional}

Depois de se tornar rotariano, João passou a se envolver nas atividades do seu clube, participando de projetos de abrangência local. Em certo momento, foi decidido que seu clube se dedicaria à realização de um projeto internacional, voltado para a organização, em conjunto com algum clube ou distrito do exterior, de uma unidade de saúde móvel a ser operada por uma organização colaboradora, proporcionando atendimento gratuito a mães e crianças em diversas comunidades. Maria, madrinha de João no Rotary, seria líder no projeto - assim como Ana e José, também integrantes do clube -, e ele prontamente colocou-se à disposição para ajudar. Ademais, por ter interesse em aprender como é a liderança em um projeto de tal tipo, inclusive para exercê-la em projetos internacionais futuros, ficou combinado que João acompanharia Maria durante o desenvolvimento do projeto.

Analisando a interação de Maria, Ana e José com os demais integrantes do grupo, João notou que, por padrão, na realização de um projeto, os líderes conseguem contar com a participação de pessoas que estão verdadeiramente comprometidas com os ideais e os objetivos a serem alcançados, ou seja, que estão efetivamente engajadas na prática do servir. Como os rotarianos são voluntários, espera-se uma dedicação natural de sua parte. Além disso, o discurso voltado para o servir por parte dos líderes, como era possível observar no projeto em questão, mostra-se como um importante incentivo. João percebeu que, com a abordagem relacionada ao servir - referência da cultura

\footnotetext{
${ }^{1}$ Relatório annual 2015-2016 do Rotary, disponível em https://my.rotary.org/document/annual-report-2015-16interactive-rotary-international-and-rotary-foundation (acesso em julho de 2017)
} 
organizacional -, os líderes unem seguidores - de variadas culturas - para a realização de projetos. Entretanto, há fatores que fazem a participação de seguidores não ocorrer ou ser reduzida no desenvolvimento de projetos. Um deles é a indisponibilidade, total ou parcial, em função de outros compromissos, a exemplo dos profissionais. A falta de conhecimento sobre as possibilidades de projetos, suas regras e sua dinâmica também é um problema. Outro fator é a falta de alinhamento com os propósitos da organização - constata-se a presença de pessoas mais interessadas no status de rotariano do que na prestação de serviços.

João também aprendeu que, na organização de um projeto, o propósito de servir deve ter alinhamento com as comunidades às quais os projetos estão relacionados. Para tanto, membros das comunidades devem estar envolvidos com os projetos, assegurando que eles atendem a importantes necessidades. Nesse sentido, rotarianos envolvidos com o projeto visitaram comunidades a serem atendidas pelo projeto para conhecer sua realidade e verificar as demandas de mães e crianças. Ademais, como relatado por Maria, a utilização de conhecimentos e habilidades de membros das comunidades, assim como de organizadores, integrantes de outros clubes e organizações parceiras, mostra-se muito útil para o êxito de um projeto. Com a experiência até então, portanto, João passou a compreender como líderes lidam com o desafio de unir seguidores - que possuem variadas referências culturais - para o propósito de realizar projetos.

\section{A busca por parceiros em um projeto internacional}

Após o mapeamento das comunidades a serem atendidas e das necessidades de mães e crianças no que diz respeito a cuidados com a saúde, a concretização de uma parceria internacional passou a ser o foco. Era necessário buscar um parceiro e, em seguida, com ele manter comunicação e desenvolver o projeto, atividades em que há interação entre pessoas de diferentes culturas sociais. A formação de parcerias entre clubes e distritos de diferentes países é fundamental para que sejam concretizados os projetos internacionais estruturados para fazer uso de subsídios globais da Fundação Rotária (a existência de parceria internacional representa uma exigência para que a Fundação Rotária colabore financeiramente com o projeto, e tal colaboração facilita a realização de projetos de grande porte). Em tal caso, encontrar parceiros representa um desafio que precisa ser superado. Com isso, os líderes direcionam esforços para a realização de parcerias, agindo diretamente e mobilizando seguidores para tentativas. Na busca por parceiros, os distritos representam o principal alvo, o que se justifica por dois motivos: (a) a equiparação financeira por parte da Fundação Rotária é maior no caso de um distrito como parceiro; e (b) um distrito normalmente conta com muito mais recursos para projetos do que um clube.

Maria relatou para João que a obtenção de parcerias tem ocorrido sobretudo em contextos de encontros pessoais. Uma vez que os rotarianos são bem-vindos às reuniões de qualquer clube, eles possuem o hábito de visitar outros clubes. Diante da necessidade de formar parcerias para projetos internacionais, há líderes que visitam clubes diversos para tratar do assunto. A principal fonte de parcerias, entretanto, tem sido a Convenção do Rotary. O evento, que é realizado anualmente, costuma contar com cerca de 30 mil pessoas, havendo representação da maioria dos distritos existentes. Assim, os líderes de projetos nele presentes têm a possibilidade de dialogar com integrantes de clubes de variados locais, incluindo líderes dos distritos. A Assembleia Internacional, evento anual voltado para o treinamento dos próximos rotarianos que atuarão como governadores isto é, que ficarão à frente dos seus distritos -, também se revela como uma oportunidade para parcerias. Em tal caso, líderes de projetos têm solicitado que os futuros governadores dos seus distritos apresentem os projetos para os demais participantes.

Maria disse para João que a busca por parceiros também acontece virtualmente. Nos grupos de discussão presentes no site do Rotary, é possível a interação entre integrantes interessados na realização de projetos. A organização também conta com o Rotary Ideas, página em que projetos que necessitam de parcerias podem ser apresentados. Contatos entre rotarianos dos mais variados clubes 
e distritos também são possibilitados pelas redes sociais - em tal caso, entretanto, é importante verificar se aqueles que se colocam como rotarianos efetivamente fazem parte da organização, e se as páginas atribuídas a clubes e distritos são de fato controladas por tais clubes e distritos.

Ainda em relação à busca por parceiros, Maria comentou com João que existem situações em alguns clubes que se mostram interessantes. Há clubes que contam com integrantes de outros países, o que tem facilitado a formação de parcerias com clubes e distritos de tais países. Há também rotarianos que, por motivos diversos, a exemplo de família e trabalho, possuem contatos em diferentes países, o que também tem proporcionado maior facilidade para a concretização de parcerias. Clubes em que tais situações não se fazem presentes, por sua vez, podem buscar sua ajuda. Outro ponto destacado por ela foi que alguns líderes têm formado parcerias baseadas em reciprocidade - um clube ou distrito aceita ser parceiro em um projeto com a condição de a parceria se repetir em um projeto de sua iniciativa. Em tal caso, vale destacar que a ideia pode ser inviabilizada pela diferença de condições financeiras entre os potenciais parceiros.

\section{Formação de parceria e interação entre os parceiros}

Como não era época de eventos, o grupo que trabalhava no projeto decidiu fazer contato com rotarianos no exterior por meio de redes sociais. Tendo em vista que Maria faria em breve uma viagem para Atlanta, nos Estados Unidos, houve conversas com rotarianos de lá. Houve negativas, em função de indisponibilidade de agenda e foco em outras áreas de atuação, mas um clube mostrou interesse em dialogar e envolver o distrito local no projeto. Assim, ficou acertado que Maria e João participariam de uma reunião do clube.

Conforme combinado, Maria e João fizeram-se presentes na reunião, que contou também com integrantes do clube e com uma representante do distrito local - Jennifer. Maria e João chegaram com antecedência ao encontro. Com isso, além de evitarem causar uma impressão ruim em relação a cumprimento de horário e uma associação a tal estereótipo, eles tiveram condições de conhecer melhor outras pessoas que também chegaram cedo e receber informações sobre o clube. Isso possibilitou uma interação mais próxima no encontro. João, pela primeira vez em uma reunião de Rotary fora do seu país, viu que o ritual seguido era idêntico ao do seu clube. Durante a reunião, Maria falou a respeito do projeto, que foi considerado relevante. Jennifer disse que o distrito poderia colaborar financeiramente com o projeto, com a destinação da verba necessária, e ficou combinado que uma comissão com três integrantes do clube - Susan, Albert e Robert - visitaria Salvador para conhecer os locais a serem beneficiados e atuar na condução do projeto. Assim, o projeto tornou-se financeiramente viável, e o trabalho com base na parceria formada seria responsável por sua concretização. João percebeu que, no que diz respeito ao estabelecimento de confiança entre as partes, o compartilhamento dos ideais da organização é um facilitador, mas os encontros pessoais em eventos e reuniões mostram-se bastante relevantes. Além disso, como comentou Maria, referências sobre os parceiros vindas de rotarianos que com eles já atuaram em conjunto também são muito úteis.

Maria falou para João que, na comunicação entre os parceiros, pessoalmente ou por conversas em vídeo, áudio ou texto, o idioma pode representar um aspecto a ser discutido, já que, em muitos projetos, os participantes não têm o hábito de falar o mesmo idioma. Em tais casos, pode-se optar pela definição de um idioma a ser utilizado - sendo comum o inglês, que é o principal idioma associado à organização - ou pelo uso de mais de um idioma nos contatos, especialmente quando há similaridade entre eles. Em um projeto no qual ela tinha participado, por exemplo, português e espanhol eram utilizados. No projeto em questão, diante da falta de conhecimento em relação ao idioma português por parte dos rotarianos do clube de Atlanta, as conversas aconteciam em inglês. $\mathrm{O}$ uso de gírias por parte dos rotarianos de Atlanta trouxe dificuldades para os diálogos no início, mas, com o passar do tempo, as dúvidas referentes às expressões utilizadas foram esclarecidas e a comunicação passou a fluir bem. 
João observou que, durante o desenvolvimento dos projetos, é essencial que os líderes, com a presença nas comunidades e o relacionamento com seus membros, conheçam seus costumes e mostrem respeito a suas referências culturais. Maria relatou que, como sempre há parceiros nos locais de realização dos projetos, é habitual que, em função do seu conhecimento e da sua experiência, eles tenham mais poder e respaldo para a tomada de decisões relacionadas às comunidade em que acontecem os projetos. Sobre a forma de tomada de decisões nos projetos, Maria disse que, sejam elas ligadas a cronograma, orçamento, responsabilidades ou outros assuntos, diferenças entre as culturas envolvidas podem fazer com que um parceiro aguarde mais tempo por uma resposta, mas é necessário o respeito a tal situação - enquanto em alguns grupos os líderes tomam decisões sem necessidade de consulta aos demais, em outros há o costume de os líderes discutirem mais os assuntos com as outras pessoas. Com a experiência acumulada, portanto, João passou a compreender como líderes lidam com o desafio de conduzir seguidores de variadas culturas rumo à realização de objetivos.

\section{Dificuldades na relação entre os parceiros}

Durante o período em que Susan, Albert e Robert estiveram em Salvador, Maria, Ana, José e João apresentaram para eles as comunidades envolvidas no projeto. Os rotarianos de Atlanta vivenciaram uma realidade diferente da que estavam acostumados a ver, e isso gerou dificuldades para o entendimento das necessidades de cuidado com a saúde de mães e crianças. Houve resistência por parte dos rotarianos de Atlanta em manter a programação do projeto, em função de não terem conhecimento sobre doenças a serem tratadas que foram listadas, o que gerou dúvidas sobre a sua relevância. Para contornar a situação, foi feita uma reunião com representantes da organização colaboradora que ficaria à frente dos atendimentos, que deram explicações sobre o assunto para Susan, Albert e Robert, os quais passaram a ter um melhor entendimento sobre o contexto das comunidades e a importância dos tratamentos a serem realizados, e aprovaram a continuidade do projeto.

João aprendeu que o conflito é inerente aos projetos internacionais no Rotary, estando relacionado a momentos de discussão, reflexão e aprimoramento. Durante o desenvolvimento de projetos internacionais, problemas e dificuldades podem surgir a qualquer instante, e os líderes devem atuar de modo que soluções para tais questões sejam alcançadas, evitando danos e buscando o aperfeiçoamento. A falta de conhecimento sobre a realidade de uma comunidade ou de alinhamento com sua cultura pode fazer com que um projeto tenha que ser reestruturado ou interrompido. Relatando sua experiência, Maria apontou que, diante de um problema ocorrido em uma região, foi organizada, por meio de um projeto, uma ampla doação de um produto para pessoas do local. Entretanto, os organizadores não se atentaram ao fato de que o produto tinha grande produção na região. Com isso, o produto não foi adquirido junto a produtores locais, mas sim trazido de outro país. O intuito de levar o produto a pessoas da região foi alcançado, mas o comércio local sofreu um sério prejuízo. Assim, foi necessária, na sequência, a adoção de medidas para revitalizar a economia da região. Em outro relato, ela disse que um projeto foi desenvolvido para possibilitar que um processo existente em uma comunidade se tornasse mais rápido, facilitando a vida dos seus membros. Contudo, as pessoas de lá não foram consultadas, e a cultura local não foi considerada. A forma de realizar o processo era um costume da comunidade, representando uma tradição há muitas gerações e um motivo de orgulho. Assim, a opção resultante do projeto foi ignorada pela comunidade, que atribuiu a ele não o significado de melhoria imaginado pelos organizadores, mas sim de desconsideração a seus hábitos.

Maria contou para João que, no relacionamento entre os parceiros, a falta de infraestrutura em alguns países pode trazer dificuldades. Em um projeto do qual ela participou, por exemplo, atrasos nas atividades estavam acontecendo em função da demora de um parceiro para responder a $e$ mails, mas isso ocorria por causa da falta de internet na cidade do parceiro. Para que as respostas 
fossem dadas, era preciso ir a uma lan house em outra cidade, distante mais de $100 \mathrm{~km}$ do local. Em situações do tipo, a compreensão mostra-se fundamental para que os projetos tenham continuação. A compreensão em relação a demora também se mostra necessária quando há falta de conhecimento em relação ao idioma adotado para a comunicação entre os parceiros, o que ela vivenciou em outro projeto - em tal caso, contava-se com o auxílio de pessoas fluentes no idioma. Ela também disse que a diferença de costumes entre os parceiros pode trazer problemas. Em um dos projetos estudados, por exemplo, enquanto um dos parceiros, seguindo um hábito ligado a seu país, produzia documentos com formalidade, o outro parceiro, de um local sem tal costume, fazia registros de maneira informal. Tendo em vista o aspecto formal dos formulários e relatórios que devem ser apresentados à Fundação Rotária, o parceiro que era caracterizado pela informalidade precisou adequar-se, adaptando sua cultura.

Continuando seus relatos, Maria falou para João que outro motivo para divergências é o aspecto financeiro. Como há restrição de recursos, são adotadas prioridades, que variam conforme os interesses dos clubes e distritos, e projetos podem ter que aguardar, por mais tempo do que o imaginado, que eles liberem verba, o que muitas vezes gera insatisfação. Em um projeto do qual ela fez parte, por exemplo, foi necessário aguardar o início de uma nova gestão para que fosse dada autorização para o uso de verba. Quando isso acontece, é importante que haja bastante transparência em relação à programação a ser seguida. Sobre as parcerias baseadas em reciprocidade, ela destacou que, no sistema eletrônico do Rotary, as autorizações para utilização de verba em um projeto independem das autorizações para utilização de verba em outro projeto. Assim, pode haver diferença significativa entre as datas das autorizações. Mais um ponto destacado por Maria foi a atenção com conflitos de interesse. Integrantes do Rotary não devem ter interesses secundários nos projetos. Em particular, não podem estar ligados ao recebimento de recursos do projeto. Qualquer impressão de que haja conflito de interesse deve ser esclarecida à Fundação Rotária. Com a experiência adquirida, portanto, João passou a compreender como líderes e seguidores de variadas culturas lidam com o desafio de conviver com suas diferenças.

\section{Concretização do projeto}

Depois da visita de Susan, Albert e Robert a Salvador, os investimentos necessários foram feitos, e o projeto foi concretizado. Os rotarianos envolvidos no projeto seguem em contato, monitorando as informações relacionadas aos atendimentos prestados pela organização parceira. E, com o aprendizado obtido ao dialogar com Maria e observar um projeto internacional se tornar realidade, João passou a se sentir preparado para exercer a liderança em projetos desse tipo.

\section{Informações complementares}

O Rotary é uma organização não governamental dedicada a trabalhos voluntários presente em mais de 200 países e regiões geográficas. Com sede em Evanston, nos Estados Unidos, o Rotary é dividido em mais de 500 distritos, contando com mais de 30 mil clubes; tais clubes reúnem mais de 1,2 milhão de integrantes, chamados de rotarianos, que ingressam na organização por meio de convite, atuam de maneira voluntária e exercem liderança local e globalmente (Rotary, 2017). O Rotary está inserido, dessa forma, em diversos contextos culturais.

O Rotary teve início em 23 de fevereiro de 1905, quando Paul Harris fundou o Rotary Club de Chicago, nos Estados Unidos, e tornou-se o primeiro presidente da organização. O nome Rotary surgiu a partir da prática inicial de rodízio das reuniões entre os escritórios de cada associado. Em 1917, o então presidente Arch Klumph criou a Fundação Rotária, fundo de dotações "com o intuito de fazer o bem no mundo". A Fundação Rotária recebe doações de rotarianos e externas, e destina os recursos para projetos desenvolvidos no Rotary (Rotary, 2017).

No Rotary, cada gestão tem início em $1^{\circ}$ de julho e término em 30 de junho, sendo tal período conhecido como ano rotário. A estrutura administrativa central, incluindo a presidência, fica na sede, 
e há também escritórios de apoio em outros locais, inclusive no Brasil, na cidade de São Paulo. Atuam, na sede e nos escritórios de apoio, mais de 800 profissionais remunerados (Rotary, 2017).

Cada distrito, que é identificado por um número, é gerido por um(a) governador(a) e sua equipe, que devem ser integrantes de clubes que fazem parte do distrito. Um dos distritos existentes é o 4550, que inclui a cidade de Salvador. Em média, um distrito conta com cerca de 60 clubes (Rotary, 2017).

Cada clube, por seu turno, é gerido por um(a) presidente e sua equipe. O nome de cada clube traz a expressão Rotary Club seguida de uma referência a sua localização. Um exemplo de clube é o Rotary Club Bahia Norte, localizado no Distrito 4550. Em média, um clube possui cerca de 40 rotarianos. Normalmente, cada clube realiza reuniões semanais com duração de uma hora (Rotary, 2017).

Em geral, os clubes atuam em suas comunidades, realizando projetos que promovam a sua melhoria e o bem-estar das pessoas. Entretanto, entre os projetos desenvolvidos no Rotary, estão incluídos os projetos internacionais organizados por meio de parcerias entre clubes e distritos de diferentes países, que envolvem a atuação em conjunto de líderes de diferentes países na organização e na coordenação (Rotary, 2017). Em tal cenário, portanto, a liderança intercultural é um fator sempre presente.

Nos projetos internacionais organizados por meio de parcerias entre clubes e distritos de diferentes países, é possível contar com subsídios globais da Fundação Rotária, que representam uma contribuição financeira da Fundação Rotária para tais projetos. Para tanto, os projetos devem atender a alguns requisitos, sendo os principais os seguintes: (a) valor mínimo de US\$ 30 mil; (b) pelo menos $30 \%$ das contribuições referentes à diferença entre o valor total do projeto e o valor do subsídio global devem vir de fora do país do projeto e do distrito anfitrião; (c) ligação com necessidades identificadas na comunidade anfitriã; (d) impacto sustentável e de longo prazo; (e) resultados mensuráveis; e (f) alinhamento com pelo menos uma das áreas de enfoque do Rotary. Apesar de sua atuação poder envolver projetos nas mais distintas áreas, o Rotary, atualmente, possui seis áreas de enfoque: paz e prevenção/resolução de conflitos; prevenção e tratamento de doenças; recursos hídricos e saneamento; saúde materno-infantil; educação básica e alfabetização; e desenvolvimento econômico e comunitário. Para se determinar o valor do subsídio global, que pode variar de US\$ 15 mil até US\$ 200 mil, tem-se que cada contribuição proveniente de distrito por meio do Fundo Distrital de Utilização Controlada (FDUC) é equiparada em 100\% pela Fundação Rotária, enquanto contribuições de clubes e externas são equiparadas em 50\% (Rotary, 2017).

Quando um projeto internacional envolvendo parceria entre clubes e distritos de diferentes países é organizado, normalmente são formados um comitê local, que conta com rotarianos da região em que o projeto será realizado, e um comitê internacional, que inclui rotarianos de fora do país. Em cada comitê, que em geral conta três pessoas, uma é designada como contato principal. Esses contatos principais, juntamente com os demais integrantes dos comitês, costumam ser os principais líderes do projeto (Rotary, 2017).

O Rotary disponibiliza em seu site um material que traz os passos e as regras dos projetos internacionais estruturados para fazer uso de subsídios globais da Fundação Rotária. Além disso, apresenta o Rotary Showcase, página em que há a exposição de muitos exemplos de projetos internacionais realizados na organização (Rotary, 2017).

A figura a seguir integra o Relatório 2015-2016 do Rotary e traz informações sobre os investimentos (em dólar) da Fundação Rotária em subsídios globais no período de $1^{\circ}$ de julho de 2015 a 30 de junho de 2016. 
PRÁTICA INTERCULTURAL DA LIDERANÇA: DESAFIOS NA ORGANIZAÇÃO ROTARY Otacílio Torres Vilas-Boas, Eduardo Davel

Figura 1. Subsídios globais de 1/7/2015 a 30/6/2016

1.165 Subsidios Globais financiaram atividades de internacionais de grande porte com resultados mensuráveis e sustentáveis nas nossas seis áreas
de enfoque $\mathbf{\$ 7}$.

() Prevençắo e tratamento de doenças 378 Subsidios ${ }^{\text {S27 }} \mathrm{M}$

Recursos hidricos e saneamento

$$
272 \mathrm{~s} 18,9 \mathrm{~m}
$$

Educaçăo básica e alfabetizaçăo 173 s $10,3 \mathrm{~m}$

Desenvolvimento econômico e comunitário 165 s9,2m

Saúde materno-infantil

$$
93{ }^{5} 6,9 \mathrm{M}
$$

Paz e prevençăo/resolução de conflitos excluindo Centros Rotary pela Paz)

$8453,7_{\mathrm{M}}$

Fonte: Reproduzido de Rotary (2017, p.3) 